\title{
Safety and Tolerability of Takeda's Bivalent Virus-Like Particle (VLP) Norovirus Vaccine (NoV) in Children
}

Taisei Masuda, Paul M Mendelman, Michael Hellwig, Jim Sherwood, Frank Baehner, Astrid Borkowski

Takeda Pharmaceuticals AG, Zurich. Switzerland

\section{OBJECTIVES}

Since the introduction of routine rotavirus immunisation noroviruses (Nov) have become

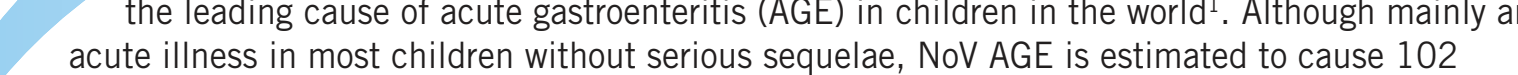
deaths $95 \% \mathrm{Cl}$ 69-139) in children under 5 years of age in Europe each year'. There is currently no Takeda is developing a bivalent norovirus vaccine (Nov) candidate consisting of two virus-like particle containing epitopes from three Gll.4 strains to provide broad coverage against the predominant circulating genotype

ne have estabished the satety, tolerability and immunogenicicity of this vaccine candidate in adultsta and we now report on the tirst interim data of the safety and tolereability

children from 1 through 8 years of age.
presentation (Abstract: ESP18-0787).

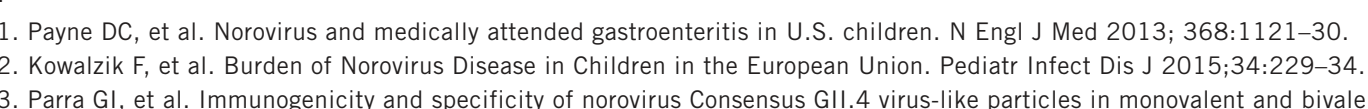

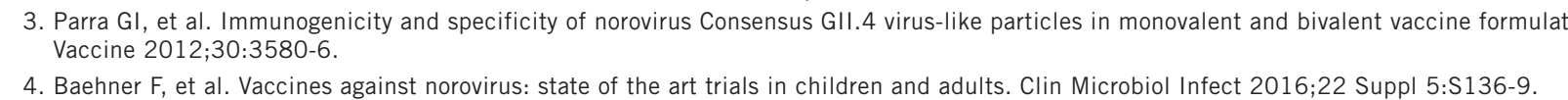

\section{PARTICIPANTS AND METHODS}

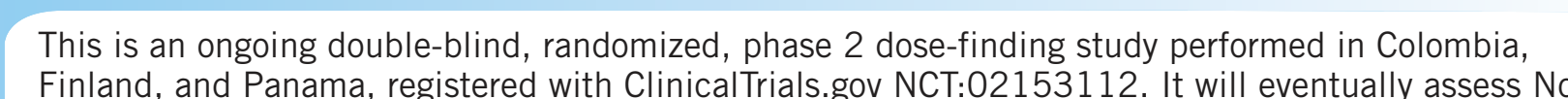

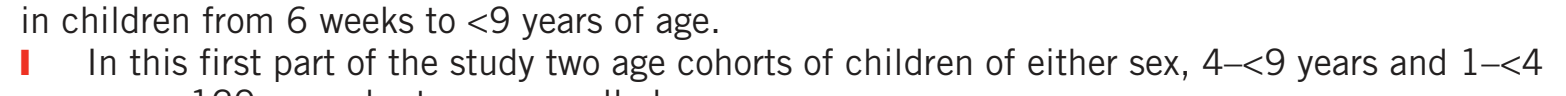

years, 120 per cohort, were enrolled.
Each cohort was randomized $1: 11: 1: 1$ into four groups to receive intramuscular injections of NoV

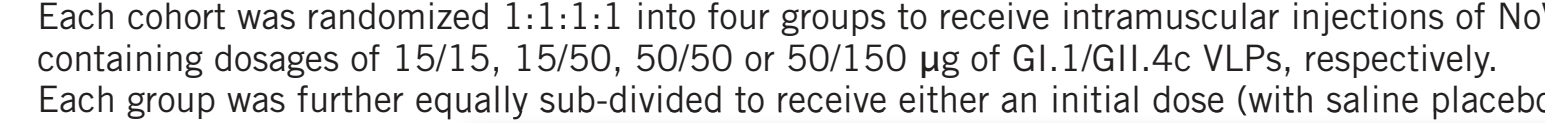

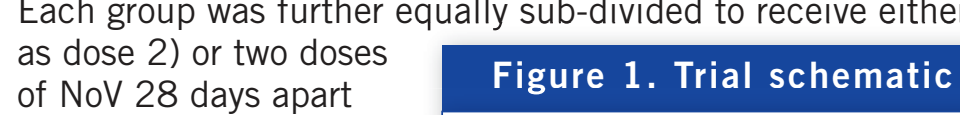

(see Figure 1, Trial

schematic).
Parents completed diary

cards for 7 days after

each waccination
(Figure 2) non which were
solicited

* local reactions:

injection site pain,
erythema, induration,

swelling
systemic a

systemic adverse
events in $4-<9$ year

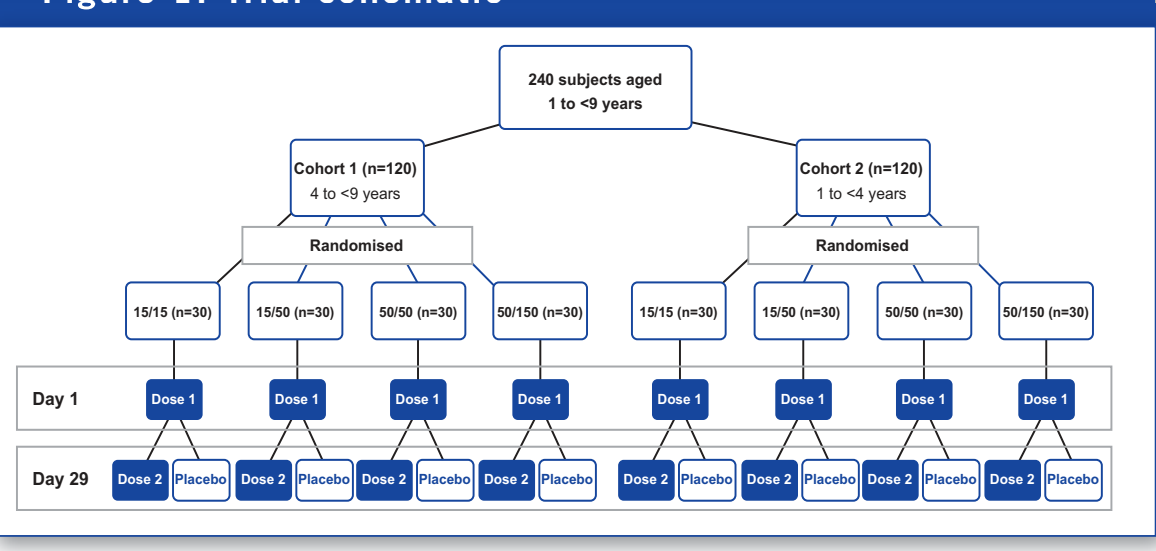

olds: headache, fatijue, myalgia, arthralgia, vomiting, diarthea
systemic adverse events in $1-<4$ year-olds: irritabilitylfussiness,

vomiting, diarthea

\section{Figure 2. Time course of study}

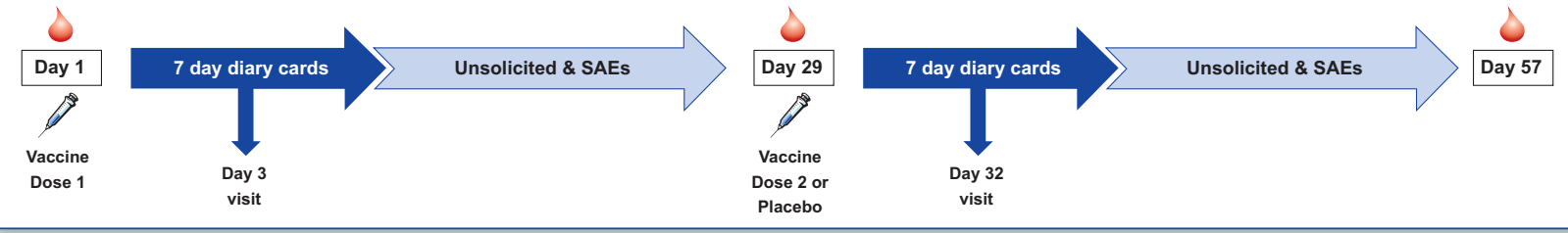

Parents recorded on the diary cards any unsolicited adverse events or serious adverse events
(SAE) until the next studuy visiti (28 days after each vaccination); these AEs were subsequently Alled adverse venents were graded tor sol

to the vaccination was assessed by the investigator.

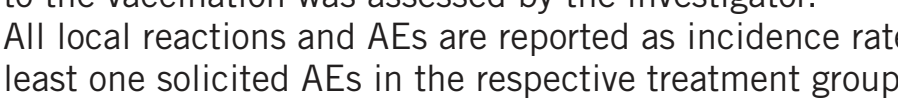

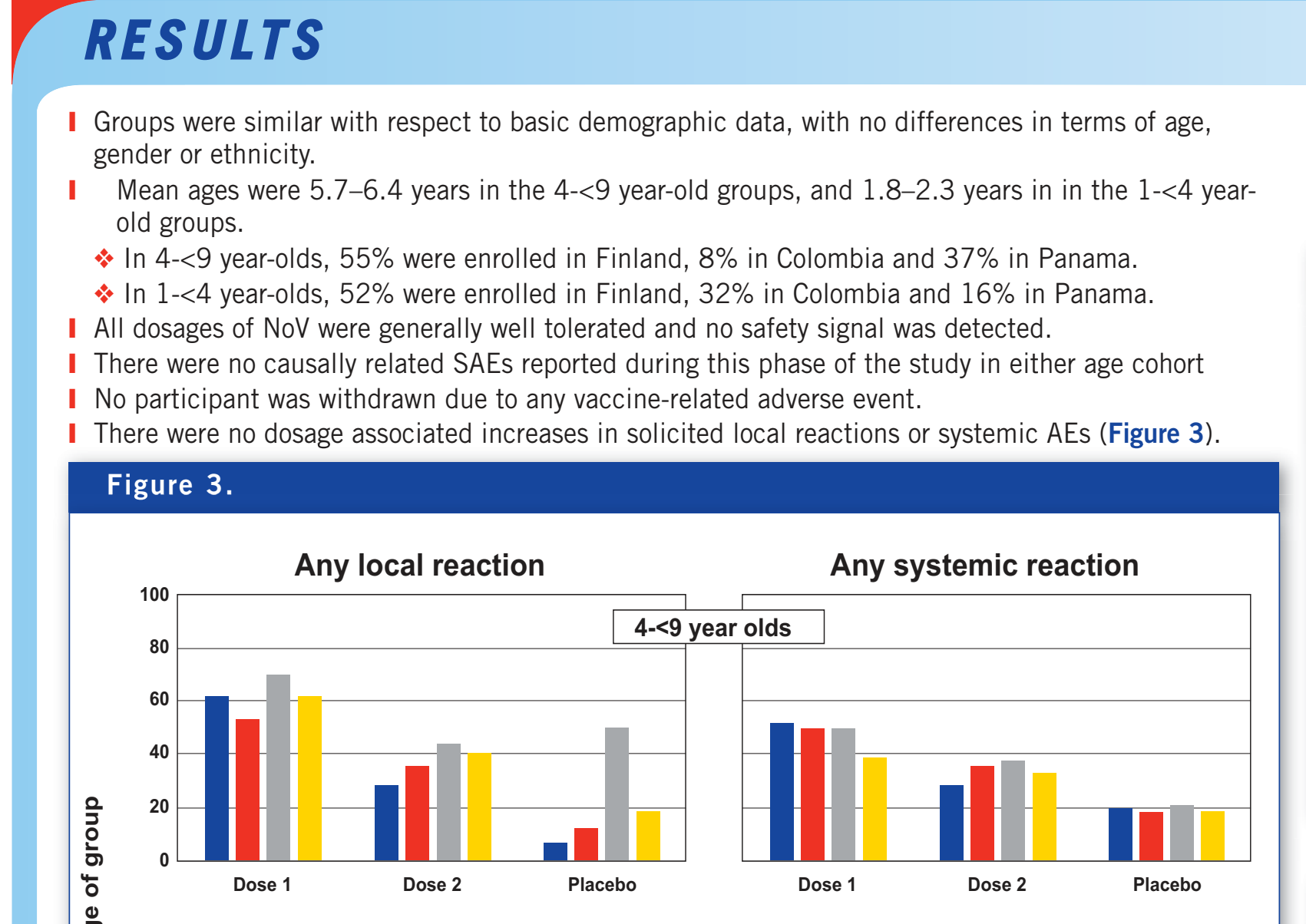

Most frequent systemic AEs were headache, fatigue and myalgia in 4- 49 year-olds (Table 1)

1- $<4$ year-olds iritability, drowsiness and loss of appetite were the most frequent AEs (Table 1$)$

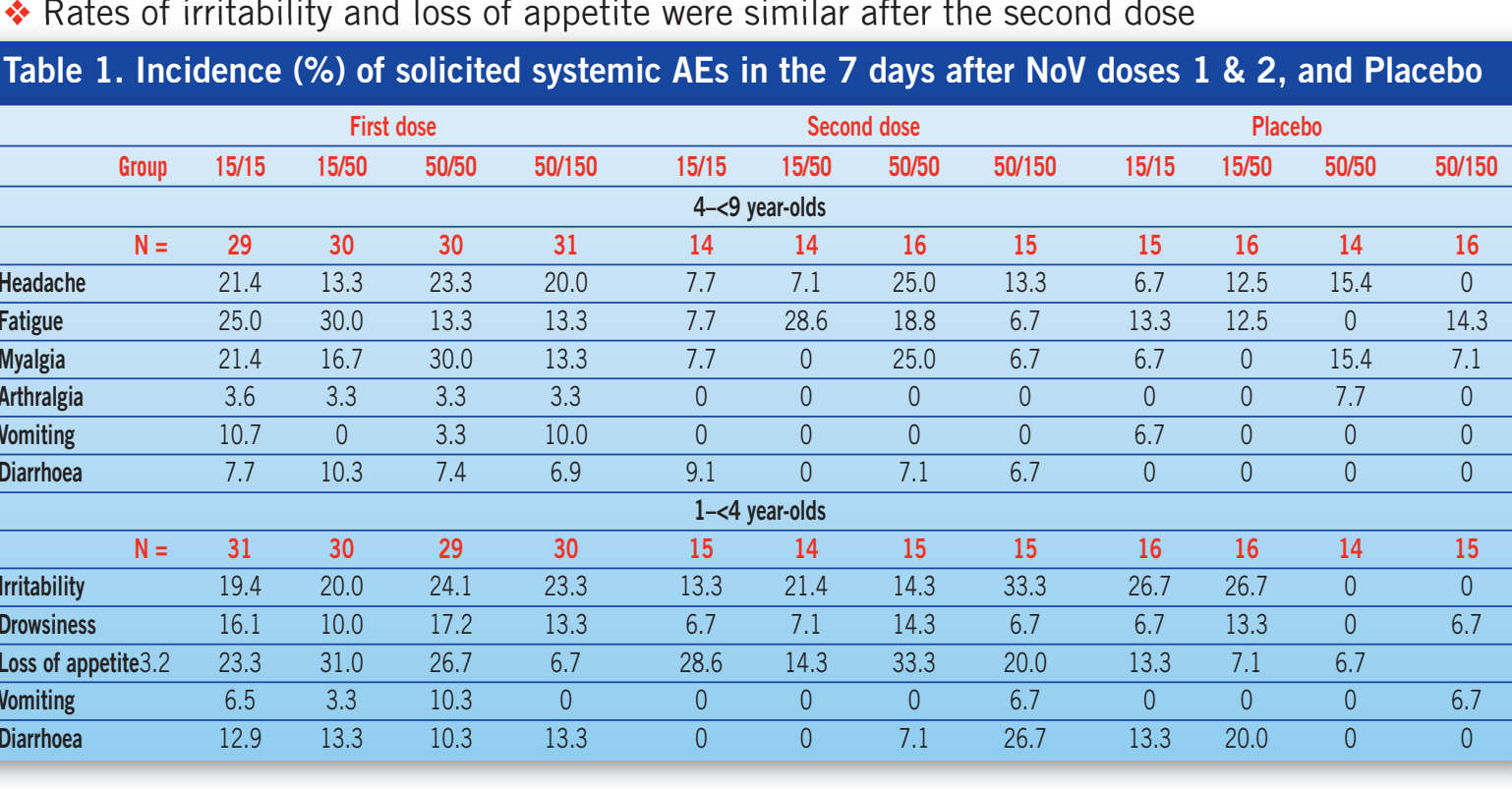

Temperature $\geq 38^{\circ} \mathrm{C}$ was infrequent in all groups in both age cohorts (Figure 5 ).

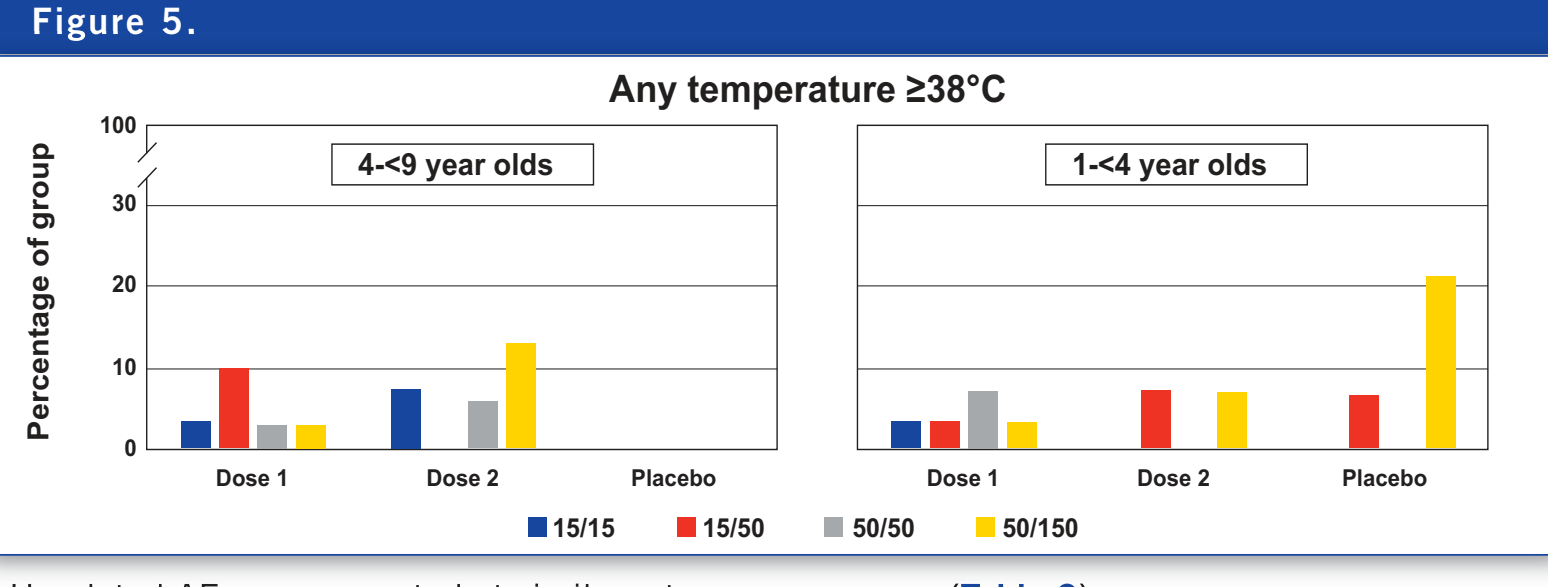

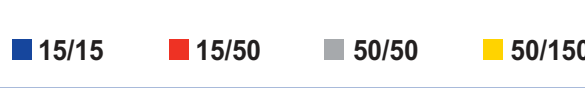

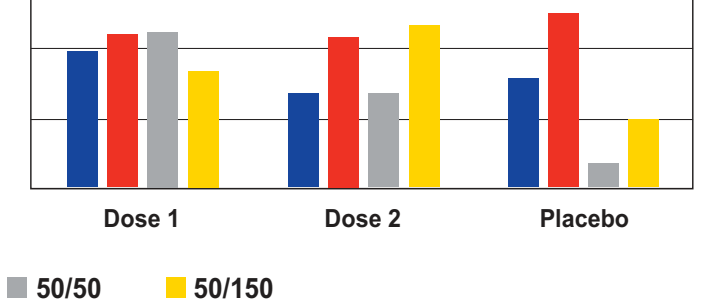

I Local reactions were more frequent in $4-<9$ year-olds $(61,7$
$1<4$ year-olds $26.7 \%$ after first dose across dosages).

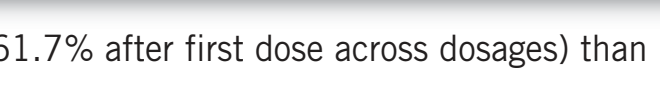

1. Systemic AEs were reported at similar rates across dosages
across dosagess and $1-<4$ year-olds $40.0 \%$ atter first dose)

1 Rates of toth local reactions and systemic AEs were lower after a second dose, and higher than
placeco in most instances.

The majority of solicited

The most frequent local reaction was injection site pain in both age cohorts (Figure 4

The majority of locat reactions were described as nild or moderate, with few described as severe.

Other local reactions

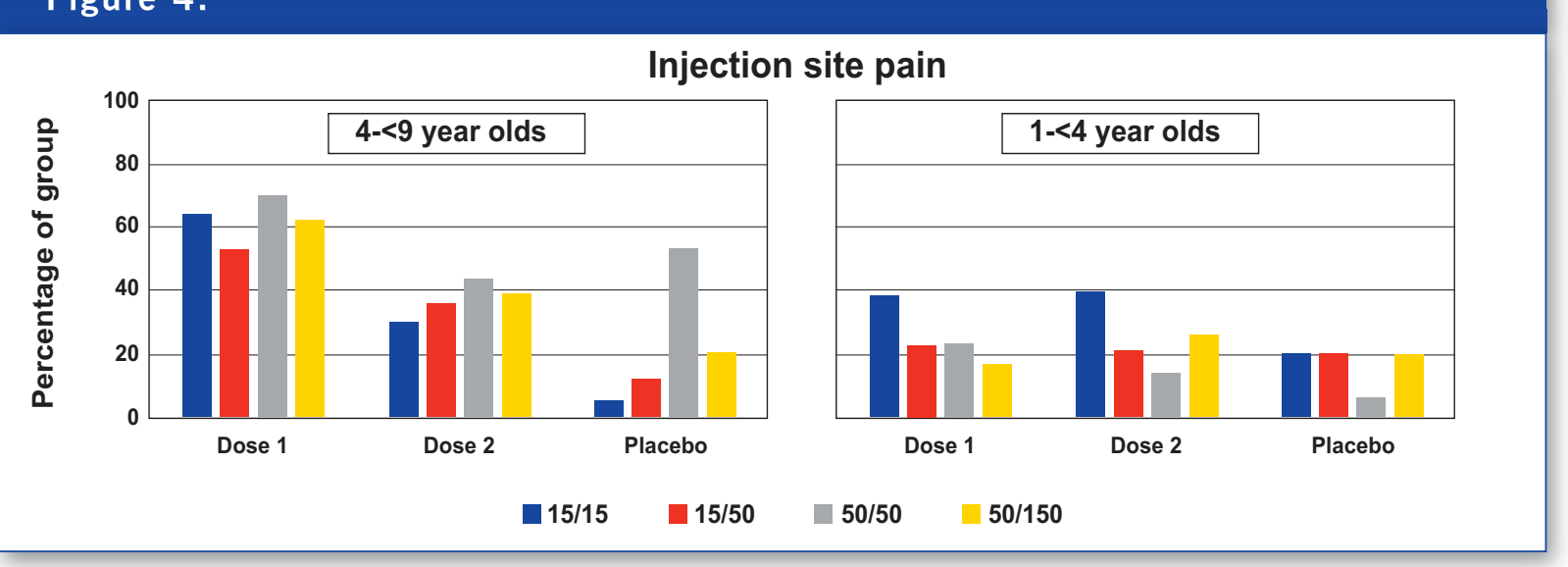

I Unrelated AEs were reported at similar rates across groups (Table 2).

Table 2. Incidence (\%) and relatedness of unsolicited AEs in the 28 days after Nov doses 1 \& 2,

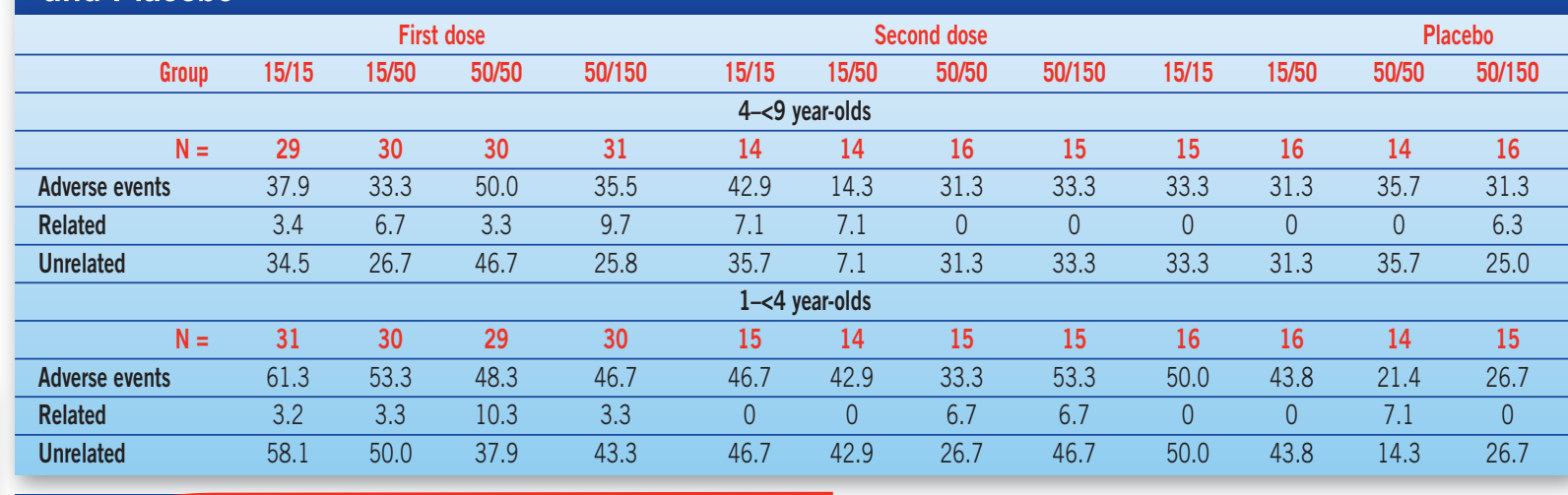

CONCLUSIONS

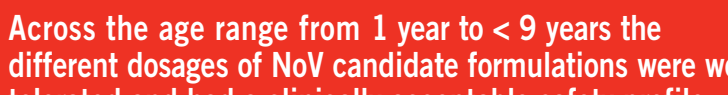

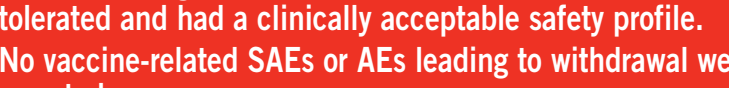

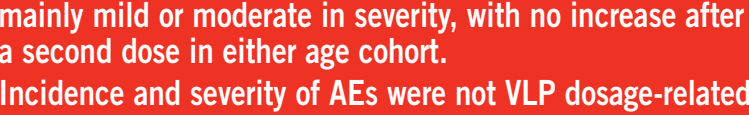

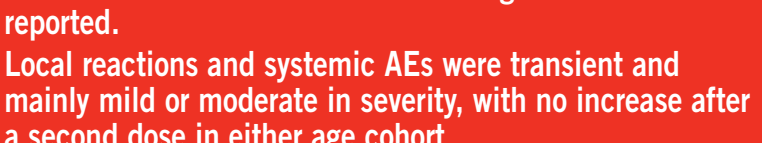

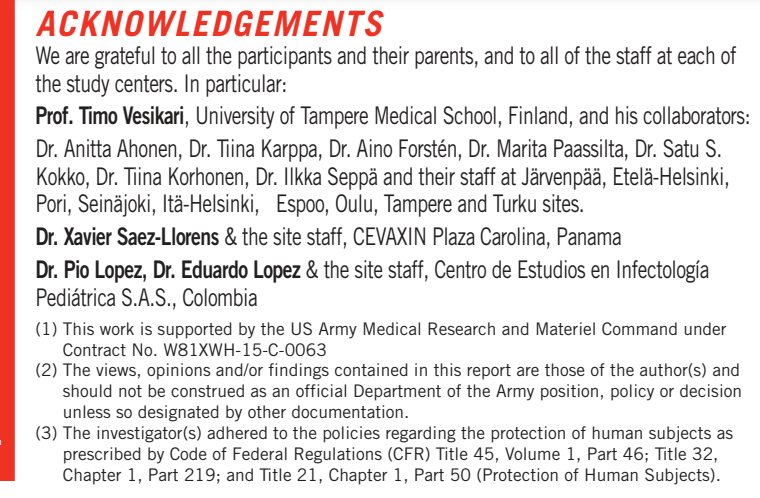

